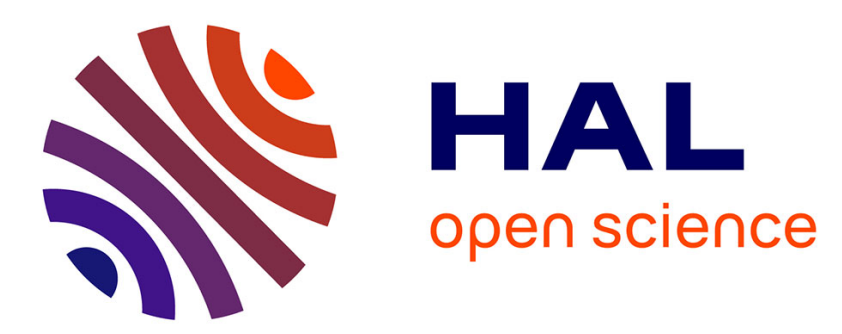

\title{
Deep Learning Techniques to Improve Intraoperative Awareness Detection from Electroencephalographic Signals
}

\author{
Oleksii Avilov, Sébastien Rimbert, Anton Popov, Laurent Bougrain
}

\section{To cite this version:}

Oleksii Avilov, Sébastien Rimbert, Anton Popov, Laurent Bougrain. Deep Learning Techniques to Improve Intraoperative Awareness Detection from Electroencephalographic Signals. IEEE Engineering in Medicine and Biology Society 2020, Jul 2020, Montreal, Canada. hal-02920320

\section{HAL Id: hal-02920320 \\ https://hal.inria.fr/hal-02920320}

Submitted on 24 Aug 2020

HAL is a multi-disciplinary open access archive for the deposit and dissemination of scientific research documents, whether they are published or not. The documents may come from teaching and research institutions in France or abroad, or from public or private research centers.
L'archive ouverte pluridisciplinaire $\mathbf{H A L}$, est destinée au dépôt et à la diffusion de documents scientifiques de niveau recherche, publiés ou non, émanant des établissements d'enseignement et de recherche français ou étrangers, des laboratoires publics ou privés. 


\title{
Deep Learning Techniques to Improve Intraoperative Awareness Detection from Electroencephalographic Signals
}

\author{
Oleksii Avilov ${ }^{1,2}$, Sébastien Rimbert ${ }^{1}$, Anton Popov $^{2}$, and Laurent Bougrain ${ }^{1}$
}

\begin{abstract}
Every year, millions of patients regain consciousness during surgery and can potentially suffer from posttraumatic disorders. We recently showed that the detection of motor activity during a median nerve stimulation from electroencephalographic (EEG) signals could be used to alert the medical staff that a patient is waking up and trying to move under general anesthesia [1], [2]. In this work, we measure the accuracy and false positive rate in detecting motor imagery of several deep learning models (EEGNet, deep convolutional network and shallow convolutional network) directly trained on filtered EEG data. We compare them with efficient non-deep approaches, namely, a linear discriminant analysis based on common spatial patterns, the minimum distance to Riemannian mean algorithm applied to covariance matrices, a logistic regression based on a tangent space projection of covariance matrices (TS+LR). The EEGNet improves significantly the classification performance comparing to other classifiers ( $p$ value $<0.01$ ); moreover it outperforms the best non-deep classifier (TS+LR) for $\mathbf{7 . 2 \%}$ of accuracy. This approach promises to improve intraoperative awareness detection during general anesthesia.
\end{abstract}

\section{INTRODUCTION}

Three hundred million of general anesthesia procedures are being performed each year all over the world [3]. Among these patients, from 0.2 to $1.3 \%$ are victims of Accidental Awareness during General Anesthesia (AAGA) [4]. Although anesthesiologists try to closely monitor patients to prevent this terrifying phenomenon, there is currently no efficient solution to accurately predict it [5].

In previous works [1], [2], we proposed the concept of an innovative passive brain-computer interface (BCI) based on the intention of movement to prevent AAGA. Indeed, patients typically try to move to alert the medical staff during an AAGA, but discover that they are unable to. The proposed solution incorporates a Median Nerve Stimulation (MNS) causing specific modulations in the motor cortex, which are known to be altered by an intention of movement. The design of such a BCI presents us with the challenge of obtaining a high level of accuracy, which would guarantee the reliability of the BCI device so that it can be used in a clinical setting. The accuracy obtained for a movement imagery (MI) versus Rest classification in the BCI field in general remains low and should be improved to create a device reliable enough to be

\footnotetext{
${ }^{1}$ Oleksii Avilov, Sébastien Rimbert and Laurent Bougrain are with the Université de Lorraine, CNRS, Inria, LORIA, F54000, Nancy, France oleksii.avilov@loria.fr, sebastien.rimberteinria.fr, laurent.bougraineloria.fr

${ }^{2}$ Oleksii Avilov and Anton Popov are with the Electronic Engineering Department, Igor Sikorsky Kyiv Polytechnic Institute, Kyiv, Ukraine anton.popoveieee.org
}

used in hospitals. Previous studies have shown that a painless stimulation of the median nerve induces an event-related desynchronization (ERD) during the stimulation while an event-related synchronization (ERS) appears after the stimulation [6]. More interestingly, a motor task performed during a MNS abolishes the patterns previously generated by this stimulation. In our study [2] it was already shown that a MI + MNS vs MNS classification results can have better accuracy than a MI vs Rest classification, suggesting that MNS could be used as a trigger. The best classification performance was reached by a logistic regression (LR) based on a tangent space (TS) projection of covariance matrices (TS+LR) which obtained $70.5 \%$ accuracy for an MI vs. Resting state task comparable to $82.03 \%$ for an MI + MNS vs. MNS task.

While deep neural networks show better performances compared to standard machine learning methods in almost all areas, analysis of EEG signals especially in the BCI domain remains an area where the use of deep learning techniques is still limited. The potential of deep learning architectures which can deal with raw EEG signals especially for motor imagery based tasks is rather unexplored and is an active research field [7].

\section{MATERIALS AND METHODS}

\section{A. Participants and Electrophysiology Data}

Sixteen right-handed healthy volunteers ( 8 females; 19 to 57 years-old; $28.56 \pm 13.3$ years old, without medical history which could influence the task) were recruited for this study. EEG signals were acquired at $2048 \mathrm{~Hz}$ using the OpenViBE platform with a Biosemi Active Two 128-channel EEG system.

Participants signed an informed consent which was approved by the ethical committee of Inria (COERLE, approval number: 2016-011/01) as it satisfied the ethical rules and principles of the institute.

\section{B. Experimental Tasks}

The aim of this research is to discriminate between two different brain activities: a motor imagery during a median nerve stimulation (MI + MNS) and a median nerve stimulation during rest (MNS). Participants were asked to perform each condition randomly during two runs of 26 trials per condition. So in total the database consists of 52 examples of each class for every subject.

Condition 1: motor imagery with a median nerve stimulation: During the MI + MNS condition (C1), subjects had to perform a motor imagination while their median nerve was stimulated 750 milliseconds after the start of the motor 


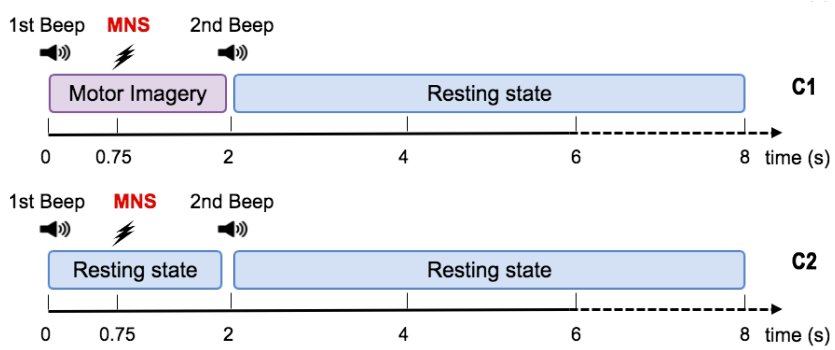

Fig. 1. Timing schemes of the trial for $\mathrm{C} 1$ and $\mathrm{C} 2$. For all motor tasks, one low frequency beep indicates when to start the task. For the MNS+MI condition, the MNS occurs at 750 milliseconds after the first beep. The end of the MI is announced by a high frequency beep and followed by a rest period of 6 seconds.

imaginary task (Fig. 1). A low frequency beep indicated when the subject had to start the motor imagery and a high frequency beep indicated when to stop it. The duration of the stimulation was $100 \mathrm{~ms}$ and stimulation intensity was adapted for each subject and varied between 8 and $15 \mathrm{~mA}$.

Condition 2: median nerve stimulation only: The MNS only condition (C2) consisted of a series of stimulations of the median nerve during rest. We placed the two electrodes of stimulation on the wrist according to the standards [6].

\section{Data Preprocessing}

At the first step, EEG signals were re-referenced to common average reference. Then EEG signals were resampled at $128 \mathrm{~Hz}$ and divided into 3 seconds trials for classification; starting at 250 milliseconds after the first beep (Fig. 1) corresponding to 384 samples ( $3 \mathrm{~s}$ at $128 \mathrm{~Hz}$ ) per channel. Then each signal was filtered in the mu+beta frequency band (8-30 Hz) using a 4th-order Butterworth band-pass filter [8] and standardized by removing the mean and scaling to unit variance using StandardScaler from Scikit-learn [9]. No artifact rejection technique was applied to the data. No data augmentation strategy was applied to the data.

\section{Feature Extraction and Classification}

In this study, we selected three deep neural network architectures to compare their performances to three well-known approaches for classification in MI-based BCI domain.

The first standard approach uses 4 common spatial pattern (CSP) filters for feature extraction [10] and a linear discriminant analysis (LDA) for classification.

The second one uses covariance matrices for feature extraction and the minimum distance to Riemannian mean algorithm (MDRM) for classification [11].

The third one uses a tangent space projection of covariance matrices for feature extraction and a Logistic Regression for classification (TS+LR) as described in [2].

Deep neural networks have been trained directly on filtered EEG data. We used i) a deep convolutional network (DeepConvNet) [12]) as reference for deep networks; ii) a shallow convolutional network (ShallowConvNet) [12] because they are designed specifically for oscillatory signal classification; and iii) a generic architecture for EEG data (EEGNet) proposed in [13] that had already obtained good results on several BCI paradigms.

Performances have been evaluated comparing the accuracy and the false positive rate (FPR) since a low FPR reduces false alarms when monitoring peroperative awereness.

DeepConvNet: DeepConvNet has four convolution-maxpooling blocks, with a special first block designed to handle the EEG input, followed by three standard convolution-maxpooling blocks and a dense softmax classification layer. The first convolutional block was split into two convolutional layers in order to better handle the large number of input channels [12]. Details about the network architecture used in this paper can be found in Table I.

ShallowConvNet: The architecture designed for the Shallow Convolutional Network was inspired by the Filter Bank Common Spatial Pattern (FBCSP) algorithm. The first two layers of the ShallowConvNet perform a temporal and a spatial convolution, as in the DeepConvNet. After the two convolutions of the ShallowConvNet, a squaring nonlinearity $x^{2}$ activation function, a mean pooling layer and a logarithmic activation function $\log (x)$ followed; together these steps are analogous to the trial log-variance computation in FBCSP [12]. Full details about the network architecture can be found in Table II.

EEGNet: The first layer of the EEGNet is a temporal convolution to learn frequency filters. Then follows a depthwise convolution layer, connected to each feature map individually to learn frequency-specific spatial filters. The separable convolution is next convolutional layer; it is a combination of a depthwise convolution, which learns a temporal summary for each feature map individually, followed by a pointwise convolution, which learns how to optimally mix the feature maps together [13]. We tested sets of different parameters (not detailed in the paper). We kept 8 temporal filters $F_{1}=8$, 2 multiplier depth $D=2$ (which determines the number of spatial filters), 16 pointwise filters $F_{2}=16$ and $(1,32)$ for the lengths of temporal kernel size for the first convolutional layer. Full details about the network architecture can be found in Table III.

Training parameters: Deep neural networks were implemented with the TensorFlow framework and Keras and trained using Grid'5000 [14] to speed up the computing. ADAM [15] is used as the optimization method. The parameters are set to default values as proposed in [15]. Categorical cross-entropy is taken as the optimization criterion. Batch size 28 was selected. We run 2000 training iterations (epochs) and perform validation stopping, saving the model weights which produced the lowest validation set loss. Usually model's best validation set performance was observed after 300-2000 epochs, what varies greatly depending on the subject data. For all neural networks we used a batch normalization [16] layer before the activation layer and a dropout layer after the activation with a probability of $50 \%$. Finally, DeepConvNet has 214,077 parameters, ShallowConvNet has 209,442 and EEGNet has 3,362 parameters. 
TABLE I

PARAMETERS OF THE DEEPCONVNet ARCHITECTURE. WHERE $C=128$ IS THE NUMBER OF CHANNELS AND $N$ IS NUMBER OF CLASSES. $T=3 \mathrm{~S} \times 128 \mathrm{~Hz}=384$ IS THE NUMBER OF TIME SAMPLES .

\begin{tabular}{l|l|l} 
Layer (Activation) & \# filters / (kernel size) & Output \\
\hline Input & & $(1, C, T)$ \\
\hline Conv2D & $25 /(1,5)$ & $(25, C, 380)$ \\
Conv2D (ELU) & $25 /(C, 1)$ & $(25,1,380)$ \\
MaxPool2D & $(1,2)$ & $(25,1,190)$ \\
\hline Conv2D (ELU) & $50 /(1,5)$ & $(50,1,186)$ \\
MaxPool2D & $(1,2)$ & $(50,1,93)$ \\
\hline Conv2D (ELU) & $100 /(1,5)$ & $(100,1,89)$ \\
MaxPool2D & $(1,2)$ & $(100,1,44)$ \\
\hline Conv2D (ELU) & $200 /(1,5)$ & $(200,1,40)$ \\
MaxPool2D & $(1,2)$ & $(200,1,20)$ \\
Flatten & & $(4000)$ \\
\hline Dense (Softmax) & $N=2$ & $(2)$
\end{tabular}

TABLE II

Parameters of the ShallowConvNet Architecture. WHERE $C=128$ IS THE NUMBER OF CHANNELS AND $N$ IS NUMBER OF CLASSES. $T=384$ IS THE NUMBER OF TIME SAMPLES

\begin{tabular}{l|l|l} 
Layer (Activation) & \# filters / (kernel size) & Output \\
\hline Input & & $(1, C, T)$ \\
\hline Conv2D & $40 /(1,13)$ & $(40, C, 372)$ \\
Conv2D & $40 /(C, 1)$ & $(40,1,372)$ \\
Activation(square) & & $(40,1,372)$ \\
AveragePool2D & $(1,35)$, stride $(1,7)$ & $(40,1,49)$ \\
Activation $($ log) & & $(40,1,49)$ \\
Flatten & & $(1960)$ \\
\hline Dense(Softmax) & $N=2$ & $(2)$
\end{tabular}

TABLE III

PARAMETERS OF THE EEGNet ARChitecture. WHERE $C=128$ IS THE NUMBER OF CHANNELS, $T=384$ IS THE NUMBER OF TIME SAMPLES, $F_{1}=8$ IS THE NUMBER OF TEMPORAL FILTERS, $D=2$ IS THE MULTIPLIER DEPTH , $F_{2}=16$ IS THE NUMBER OF POINTWISE FILTERS AND $N=2$ IS THE NUMBER OF CLASSES.

\begin{tabular}{l|l|l} 
Layer (Activation) & \# filters / (kernel size) & Output \\
\hline Input & & $(1, C, T)$ \\
\hline Conv2D & $F 1 /(1,32)$ & $(F 1, C, T)$ \\
\hline DepthwiseConv2D (ELU) & $D * F 1 /(C, 1)$ & $(D * F 1,1, T)$ \\
AveragePool2D & $(1,4)$ & $(D * F 1,1, T / 4)$ \\
\hline SeparableConv2D (ELU) & $F 2 /(1,16)$ & $(F 2,1, T / 4)$ \\
AveragePool2D & $(1,8)$ & $(F 2,1, T / 32)$ \\
Flatten & & $(F 2 * T / 32)$ \\
\hline Dense(Softmax) & $N$ & $(N)$
\end{tabular}

\section{E. Data analysis}

We report subject-specific classification results corresponding to a ten-fold blockwise cross-validation, where eight of the ten blocks are chosen as the training set, one block as the validation set, and the remaining block as the test set. We performed a Wilcoxon signed-rank test to show the significant difference about accuracy obtained by different models.

\section{RESULTS}

For each classifier, accuracy and false positive rate of a MI + MNS vs. MNS discrimination task were averaged for a ten fold estimation and for all subjects. EEGNet has the highest performance according to both accuracy (91.6\%) and false positive rate $(8.8 \%)$. Boxplots showing the distribution of classification accuracy and FPR are represented on Fig. 2 and Fig. 3 respectively. Results obtained by ShallowConvNet are comparable to results obtained by the best standard model i.e. here TS+LR. The Wilcoxon signed-rank test showed highly significant differences between performances obtained by EEGNet and each other model both for accuracy ( $p$-value $<0.005)$ and false positive rate $(p$-value $<0.01)$.

Individual classification results show a higher classification performance with EEGNet for 12 subjects (Fig.4).

\section{DISCUSSION AND CONCLUSIONS}

In this study we have shown the ability of deep learning techniques to extract features from filtered EEG signals for detecting intraoperative awareness. We proposed three different deep learning architectures: DeepConvNet, ShallowConvNet and EEGNet. ShallowConvNet and EEGNet shown high performances especially for the false positive rate metric which is very important when detecting intraoperative awareness, as false alarms are extremely undesirable in this task and as regain of consciousness can be potentially detected every five seconds in our procedure. Statistical tests show that EEGNet significantly outperformed other methods in terms of accuracy, false positive rate and convergence time due to its smaller number of parameters which helps to avoid overfitting.

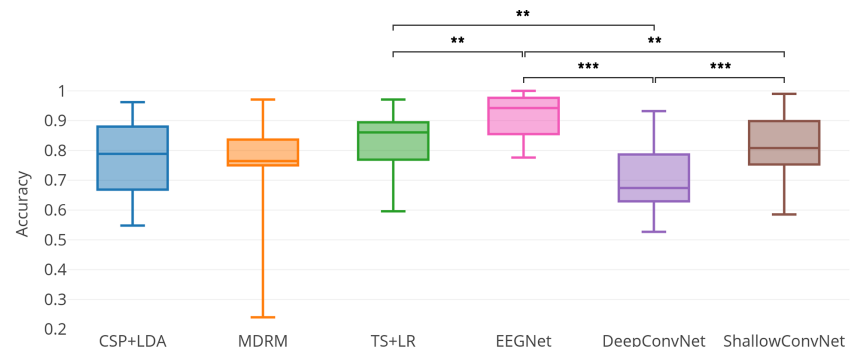

Fig. 2. Boxplots showing the distribution of classification accuracy for each model, averaged over all folds $(k=10)$ and all subjects $(n=16)$. Significance levels of Wilcoxon signed-rank test comparisons $* * p<0.01$, $* * * p<0.001$. Significance levels with other non-deep methods than TS+LR are not reported.

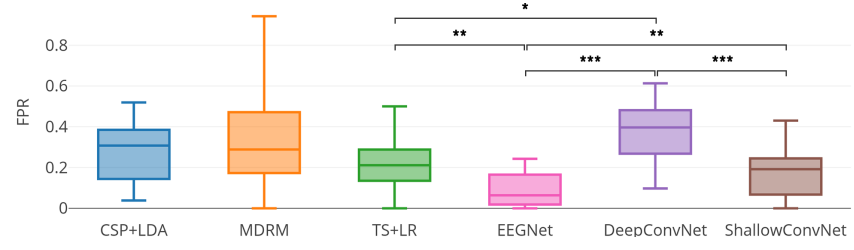

Fig. 3. Boxplots showing the distribution of false positive rate for each model, averaged over all folds $(k=10)$ and all subjects $(n=16)$. Significance levels of Wilcoxon signed-rank test comparisons $* p<0.05$, $* * p<0.01, * * * p<0.001$. Significance levels with other non-deep methods than TS+LR are not reported. 


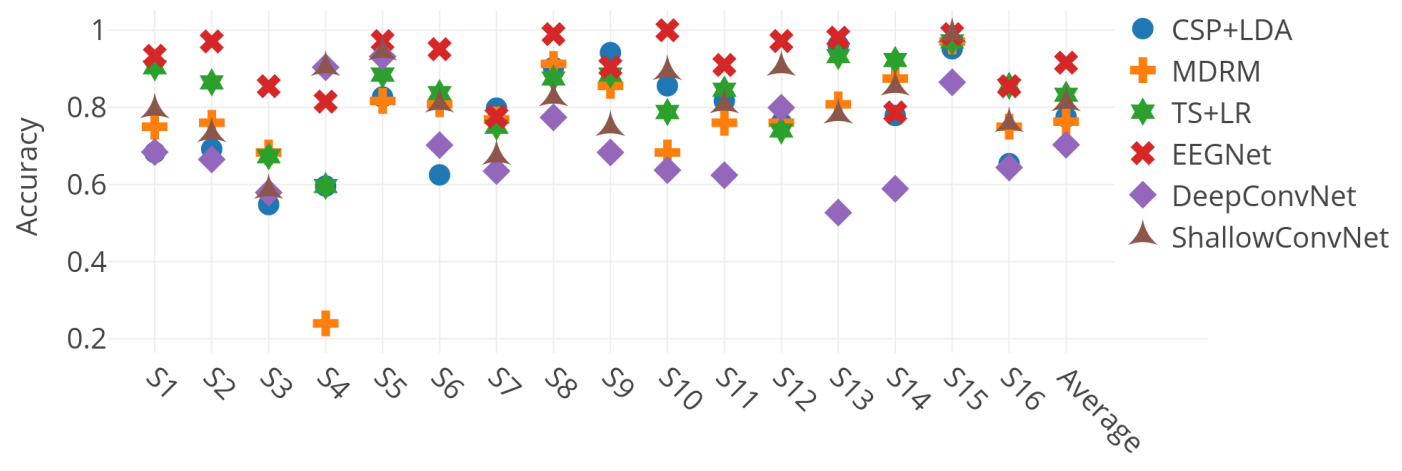

Fig. 4. Ten-fold subject-specific classification accuracies obtained for all subjects $(n=16)$ by each model for MNS vs. MI + MNS task

Although most anesthetics reduce the global metabolic activity of the central nervous system by decreasing neuronal excitability [17], the effect of anesthetics on the sensorimotor cortex appears to be relatively well preserved [18], [1]. In fact, regions of the cortex would still be receptive to information, but their ability to communicate with other regions seems to be damaged during general anaesthesia [19], suggesting that our method can be used in patients with clinical conditions [1].

Further research should be aimed at reducing the number of EEG electrodes since each electrode increases the preparation time before surgery In real conditions we have no training patterns of intention of movement. So further investigations will be aimed at subject to subject transfer learning and generative adversarial networks.

\section{ACKNOWLEDGMENT}

This research was supported by the French Embassy in Ukraine. Experiments presented in this paper were carried out using the Grid'5000 testbed, supported by a scientific interest group hosted by Inria and including CNRS, RENATER and several Universities as well as other organizations (see https://www.grid5000.fr).

\section{REFERENCES}

[1] S. Rimbert, P. Guerci, N. Gayraud, C. Meistelman, and L. Bougrain, "Innovative Brain-Computer Interface based on motor cortex activity to detect accidental awareness during general anesthesia," in IEEE SMC 2019 - IEEE International Conference on Systems, Man, and Cybernetics, Bari, Italy, Oct. 2019.

[2] S. Rimbert, P. Riff, N. Gayraud, D. Schmartz, and L. Bougrain, "Median Nerve Stimulation Based BCI: A New Approach to Detect Intraoperative Awareness During General Anesthesia," Frontiers in Neuroscience, vol. 13, p. 622, June 2019.

[3] T. Weiser, A. Haynes, G. Molina, S. Lipsitz, M. Esquivel, T. uribe Leitz, R. Fu, T. Azad, T. Chao, W. Berry, and A. Gawande, "Size and distribution of the global volume of surgery in 2012," Bull World Health Organ, vol. 94, no. 3, pp. 201-209, 2016.

[4] G. Mashour and M. Avidan, "Intraoperative awareness: controversies and non-controversies," British Journal of Anaesthesia, vol. 115, pp. 20-26, 2015.

[5] D. Almeida, "Awake and unable to move: what can perioperative practitioners do to avoid accidental awareness under general anesthesia?" J Perioper Pract, vol. 25, no. 12, pp. 257-261, 2015.

[6] A. Schnitzler, S. Salenius, R. Salmelin, V. Jousmaki, and R. Hari, "Involvement of primary motor cortex in motor imagery: a neuromagnetic study." Neuroimage, vol. 6, no. 3, pp. 201-208, Oct 1997.
[7] A. Craik, Y. He, and J. L. Contreras-Vidal, "Deep learning for electroencephalogram (EEG) classification tasks: a review," Journal of Neural Engineering, vol. 16, no. 3, p. 031001, June 2019.

[8] S. Butterworth, "On the Theory of Filter Amplifiers," In Wireless Engineer (also called Experimental Wireless and the Wireless Engineer), vol. 7, p. 536-541, 1930.

[9] F. Pedregosa, G. Varoquaux, A. Gramfort, V. Michel, B. Thirion, O. Grisel, M. Blondel, P. Prettenhofer, R. Weiss, V. Dubourg, J. Vanderplas, A. Passos, D. Cournapeau, M. Brucher, M. Perrot, and E. Duchesnay, "Scikit-learn: Machine learning in Python," Journal of Machine Learning Research, vol. 12, pp. 2825-2830, 2011.

[10] Y. Wang, S. Gao, and X. Gao, "Common Spatial Pattern Method for Channel Selelction in Motor Imagery Based Brain-computer Interface," Conference proceedings: ... Annual International Conference of the IEEE Engineering in Medicine and Biology Society. IEEE Engineering in Medicine and Biology Society. Annual Conference, vol. 5, pp. 5392-5395, 2005.

[11] A. Barachant, S. Bonnet, M. Congedo, and C. Jutten, "Multiclass Brain-Computer Interface Classification by Riemannian Geometry," IEEE Transactions on Biomedical Engineering, vol. 59, no. 4, pp. 920-928, Mar. 2012.

[12] R. T. Schirrmeister, J. T. Springenberg, L. D. J. Fiederer, M. Glasstetter, K. Eggensperger, M. Tangermann, F. Hutter, W. Burgard, and T. Ball, "Deep learning with convolutional neural networks for EEG decoding and visualization," Human Brain Mapping, vol. 38, no. 11, pp. 5391-5420, Nov. 2017, arXiv: 1703.05051.

[13] V. J. Lawhern, A. J. Solon, N. R. Waytowich, S. M. Gordon, C. P. Hung, and B. J. Lance, "EEGNet: a compact convolutional neural network for EEG-based brain-computer interfaces," Journal of Neural Engineering, vol. 15, no. 5, p. 056013, July 2018.

[14] D. Balouek, A. Carpen Amarie, G. Charrier, F. Desprez, E. Jeannot, E. Jeanvoine, A. Lèbre, D. Margery, N. Niclausse, L. Nussbaum, O. Richard, C. Pérez, F. Quesnel, C. Rohr, and L. Sarzyniec, "Adding virtualization capabilities to the Grid'5000 testbed," in Cloud Computing and Services Science, ser. Communications in Computer and Information Science, I. I. Ivanov, M. van Sinderen, F. Leymann, and T. Shan, Eds. Springer International Publishing, 2013, vol. 367, pp. 3-20.

[15] D. P. Kingma and J. Ba, "Adam: A Method for Stochastic Optimization," arXiv:1412.6980 [cs], Dec. 2014, arXiv: 1412.6980.

[16] S. Ioffe and C. Szegedy, "Batch Normalization: Accelerating Deep Network Training by Reducing Internal Covariate Shift," arXiv:1502.03167 [cs], Mar. 2015, arXiv: 1502.03167.

[17] H. Hentschke, A. Raz, B. M. Krause, C. A. Murphy, and M. I. Banks, "Disruption of cortical network activity by the general anaesthetic isoflurane," British Journal of Anaesthesia, vol. 119, no. 4, pp. 685696, Oct. 2017.

[18] Y. Blokland, J. Farquhar, J. Lerou, J. Mourisse, G. J. Scheffer, G.-J. van Geffen, L. Spyrou, and J. Bruhn, "Decoding motor responses from the eeg during altered states of consciousness induced by propofol," Journal of Neural Engineering, vol. 13, no. 2, p. 026014, 2016.

[19] S. J. Peltier, C. Kerssens, S. B. Hamann, P. S. Sebel, M. Byas-Smith, and $\mathrm{X}$. Hu, "Functional connectivity changes with concentration of sevoflurane anesthesia," NeuroReport, vol. 16, no. 3, pp. 285-288, Feb. 2005. 